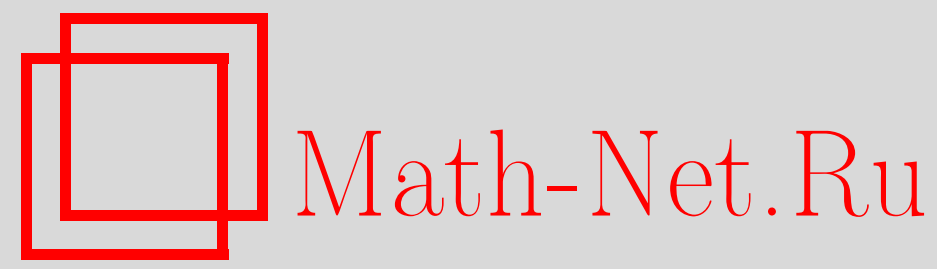

В. М. Круглов, Слабая компактность случайных сумм независимых случайных величин, Теория вероятн. и ее примен., 1998, том 43, выпуск 2, 248-271

DOI: https://doi.org/10.4213/tvp1464

Использование Общероссийского математического портала Math-Net.Ru подразумевает, что вы прочитали и согласны с пользовательским соглашением http://www. mathnet.ru/rus/agreement

Параметры загрузки:

IP : 54.224 .135 .184

26 апреля 2023 г., 10:46:32

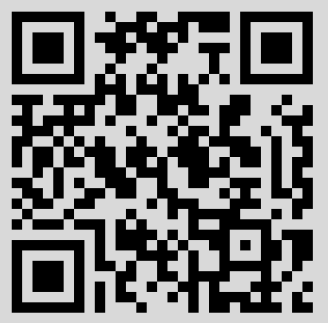


(C) $1998 \mathrm{r}$.

КРУГЛОВ В. м. $^{*}$

\section{СЛАБАЯ КОМПАКТНОСТЬ СЛУЧАЙНЫХ СУММ НЕЗАВИСИМЫХ СЛУЧАЙНЫХ ВЕЛИЧИН ${ }^{1)}$}

Исследуется сдвиг-компактность случайных сумм $S_{N_{n}}^{(n)}, S_{k}^{(n)}=$ $X_{n, 1}+\cdots+X_{n, k}$, независимых случайных величин со случайным числом слагаемых $N_{n}$ в предположении, что в каждой сумме слагаемые и их число независимы и что слагаемые удовлетворяют условию равномерной предельной малости:

$$
\max _{1 \leqslant k \leqslant N_{n}} \mathbf{P}\left\{\left|X_{n, k}\right| \geqslant \varepsilon\right\} \longrightarrow 0
$$

по вероятности для любого $\varepsilon>0$. Приведен ряд необходимых и достаточных условий для слабой компактности случайных сумм $S_{N_{n}}^{(n)}-A_{n}$ и указан вид центрирующих постоянных $A_{n}$.

Ключевые слова и фразы: случайная величина, функция распределения, слабая сходимость, слабая компактность, сдвиг-компактность, случайная сумма.

\section{1. Формулировки утверждений}

В настоящей статье приводится ряд необходимых и достаточных условий сдвиг-компактности последовательностей случайных сумм независимых случайных величин, при условии независимости числа слагаемых и самих слагаемых в каждой сумме. Из приводимых ниже теорем видно, что слабую компактность последовательности случайных сумм равномерно предельно малых независимых случайных величин можно выразить в терминах слабой компактности последовательностей центрированных сумм тех же самых случайных величин с неслучайным числом слагаемых и случайных сумм с неслучайными слагаемыми. Таким образом, общая задача о слабой компактности последовательностей случайных сумм упроцается, так как аналогичная задача для сумм независимых равномерно малых случайных величин с неслучайным числох

*Факультет вычислительной математики и кибернетики, МГУ, Воробъевы го ры, 119899 Москва, Россия.

1) Работа выполнена при поддержке Российского фонда фундаментальных, ис следований, гранты № 96-01-01919, 96-01-01920 и INTAS-RFBR грант № 95-00-95. 
слагаемых решена (см., например, лемму 2 настоящей статьи). Теорема 3 представляет собой аналог критерия сдвиг-компактности для последовательностей сумм независимых равномерно малых случайных величин с неслучайным числом слагаемых. В отношении случайных сумм с одинаково распределенными слагаемыми теоремы 5 и 6 , по всей видимости, представляют собой наиболее законченные результаты.

Задача о сдвиг-компактности и, в частности, о слабой компактности последовательностей случайных сумм была предметом ряда специальных исследований. Например, в [4] и [8] приведены необходимые и достаточные условия для слабой компактности последовательностей случайных сумм, центрированных случайными величинами, а монография [2] и статьи [5], [7] содержат ряд критериев для слабой компактности последовательностей нецентрированных случайных сумм. Статья [6] содержит некоторые необходимые и достаточные условия для последовательностей случайных сумм, центрированных константами. Наша теорема 5 по своей форме не отличается от теоремы 2 из [7] и обе эти теоремы представляют собой обобщение теоремы 1 из [5]. В теореме 5 и теореме 2 из [7] фигурирует последовательность натуральных чисел $\left\{k_{n}\right\}_{n \geqslant 1}$, обладающая специальными свойствами. Отличие этих теорем состоит в том, что в [7] утверждается существование последовательности $\left\{k_{n}\right\}_{n \geqslant 1}$, а мы предлагаем явную конструкцию построения чисел $k_{n}$. Кроме того, доказательство теоремы 5 пропе и короче, чем доказательство теоремы 2 из [7]. Наконец, незначительное изменение метода доказательства теоремы 5 позволяет доказать новую более обшую теорему 6 , содержащую необходимые и достаточные условия слабой компактности последовательности случайных сумм, центрированных константамж.

Мы будем предполагать, что все рассматриваемые случайные величины определены на вероятностном пространстве $(\Omega, \mathscr{A}, \mathbf{P})$. Условимся говорить, что последовательность случайных величин $\left\{W_{n}\right\}_{n \geqslant 1}$ слабо сходится, если последовательность функший распределения $\left\{F_{n}\right\}_{n \geqslant 1}$, $F_{n}(x)=\mathbf{P}\left\{W_{n}<x\right\}$, слабо сходится к некоторой функции распределения. Последовательность случайных величин $\left\{W_{n}\right\}_{n \geqslant 1}$ называется слабо компактной, если каждая подпоследовательность последовательности функций распределения $\left\{F_{n}\right\}_{n \geqslant 1}$ содержит свою подпоследовательность, которая слабо сходится к некоторой функции распределения. В соответствии с известным критерием $([1, \S 9$, теорема 3$])$ последовательность случайных величин $\left\{W_{n}\right\}_{n \geqslant 1}$ слабо компактна тогда и только тогда, когда выполняется условие

$$
\lim _{x \rightarrow \infty} \sup _{n} \mathbf{P}\left\{\left|W_{n}\right| \geqslant x\right\}=0
$$

Записи $F_{n} \Longrightarrow F$ (или $\left.\mathbf{P}\left\{W_{n}<x\right\} \Longrightarrow F(x)\right)$ и $W_{n} \stackrel{\mathbf{p}}{\longrightarrow} W$ будут обозначать, что последовательность функций распределения $\left\{F_{n}\right\}_{n \geqslant 1}$ слабо сходится к функции распределения $F$ и последовательность случайных 
величин $\left\{W_{n}\right\}_{n \geqslant 1}$ сходится по вероятности к случайной величине $W$. Обозначение $X \stackrel{\mathrm{d}}{=} Y$ будет использоваться, чтобы подчеркнуть, что случайные величины $X$ и $Y$ одинаково распределены.

Пусть для каждого $n=1,2, \ldots$ даны независимые случайные величины $N_{n}, X_{n, k}, k=1,2, \ldots$, причем $N_{n}$ принимает натуральные значения. Обозначим $l_{n}(q)$ левую $q$-квантиль, $q \in(0,1)$, случайной величины $N_{n}$, определение левой квантили дано перед формулировкой леммы 4 ,

$S_{k}^{(n)}=X_{n, 1}+X_{n, 2}+\cdots+X_{n, k}, \quad \alpha_{n, k}=\alpha_{n, k}(v)=\int_{|x|<v} x d \mathbf{P}\left\{X_{n, k}<x\right\}$, где $v$ - произвольное положительное число.

Теорема 1. Предположим, ито выполняется условие

$$
\sup _{1 \leqslant k \leqslant N_{n}} \mathbf{P}\left\{\left|X_{n, k}\right| \geqslant \varepsilon\right\} \stackrel{\mathrm{p}}{\rightarrow} 0 \quad \forall \varepsilon>0 \text { npu } n \rightarrow \infty .
$$

Дая того чтобы при некотором подборе вещественньх чисел $A_{n}$ последовательность случайных сумм $\left\{S_{N_{n}}^{(n)}-A_{n}\right\}_{n \geqslant 1}$ была слабо компактной, необходимо и достаточно, чтобы выполнялось одно из следующих двух условий:

А) для почти всех по мере Лебеаа $q \in(0,1)$ последовательности случайных величин

$$
\left\{\sum_{k=1}^{l_{n}(q)}\left(X_{n, k}-\alpha_{n, k}\right)\right\}_{n \geqslant 1} u\left\{\sum_{k=1}^{N_{n}} \alpha_{n, k}-\operatorname{med}\left(\sum_{k=1}^{N_{n}} \alpha_{n, k}\right)\right\}_{n \geqslant 1}
$$

слабо компактны,

В) последовательности случайных величин

$$
\left\{\sum_{k=1}^{N_{n}}\left(X_{n, k}-\alpha_{n, k}\right)\right\}_{n \geqslant 1} u\left\{\sum_{k=1}^{N_{n}} \alpha_{n, k}-\operatorname{med}\left(\sum_{k=1}^{N_{n}} \alpha_{n, k}\right)\right\}_{n \geqslant 1}
$$

слабо компактны.

Постоянные $A_{n}$ можно выбрать по формуле

$$
A_{n}=C_{n}+\operatorname{med}\left(\sum_{k=1}^{N_{n}} \alpha_{n, k}\right), \quad n=1,2, \ldots,
$$

где $C_{n}$ - любые вещественные числа, удовлетворяющие условию $\sup _{n}\left|C_{n}\right|<\infty$.

Возможно стоит отметить, что в следующем замечании предположение (2) о равномерной предельной малости случайных величин не используется.

3 а м е ч а н и е 1 . Для того чтобы при некотором подборе вешественных чисел $A_{n}$ последовательность случайных сумм $\left\{S_{N_{n}}^{(n)}-A_{n}\right\}_{n \geqslant 1}$ 
была слабо компактной, достаточно, чтобы для почти всех по мере Лебега $q \in(0,1)$ последовательность случайных величин

$$
\left\{\sum_{k=1}^{l_{n}(q)}\left(X_{n, k}-\alpha_{n, k}\right)\right\}_{n \geqslant 1}
$$

была слабо компактной и числовая последовательность

$$
\left\{\sum_{k=1}^{l_{n}(q)} \alpha_{n, k}-\operatorname{med}\left(\sum_{k=1}^{N_{n}} \alpha_{n, k}\right)\right\}_{n \geqslant 1}
$$

была ограниченной.

Постоянные $A_{n}$ можно выбрать по формуле, указанной в теореме 1.

Следующая теорема является аналогом теоремы 1 для того случая, когда условие (2), означаюшее предельную равномерную малость случайных величин $X_{n, k}, 1 \leqslant k \leqslant N_{n}$, заменяется условием предельного постоянства этих случайных величин. Обозначим $m_{n, k}$ медиану случайной величины $X_{n, k}$,

$$
\beta_{n, k} \neq \beta_{n, k}(v)=\int_{|x|<v} x d \mathbf{P}\left\{X_{n, k}-m_{n, k}<x\right\}
$$

где $v$ - произвольное положительное число.

Теорема 2. Предположим, что выполняется условие

$$
\sup _{1 \leqslant k \leqslant N_{n}} \mathbf{P}\left\{\left|X_{n, k}-m_{n, k}\right| \geqslant \varepsilon\right\} \stackrel{\mathrm{p}}{\longrightarrow} 0 \quad \forall \varepsilon>0 \text { npu } n \rightarrow \infty
$$

Дая того чтобы при некотором подборе вещественных чисел $A_{n}$ последовательность случайных сумм $\left\{S_{N_{n}}^{(n)}-A_{n}\right\}_{n \geqslant 1}$ была слабо компактной, необходимо и достаточно, чтобы выполнялось одно из следующих дв ух условий:

А) для почти всех по мере Лебега $q \in(0,1)$ последовательности случайных величин

$$
\begin{aligned}
& \left\{\sum_{k=1}^{l_{n}(q)}\left(X_{n, k}-m_{n, k}-\beta_{n, k}\right)\right\}_{n \geqslant 1} u \\
& \left\{\sum_{k=1}^{N_{n}}\left(m_{n, k}+\beta_{n, k}\right)-\operatorname{med}\left(\sum_{k=1}^{N_{n}}\left(m_{n, k}+\beta_{n, k}\right)\right)\right\}_{n \geqslant 1}
\end{aligned}
$$

слабо компактны, 
В) последовательности случайных величин

$$
\begin{aligned}
& \left\{\sum_{k=1}^{N_{n}}\left(X_{n, k}-m_{n, k}-\beta_{n, k}\right)\right\}_{n \geqslant 1} u \\
& \left\{\sum_{k=1}^{N_{n}}\left(m_{n, k}+\beta_{n, k}\right)-\operatorname{med}\left(\sum_{k=1}^{N_{n}}\left(m_{n, k}+\beta_{n, k}\right)\right)\right\}_{n \geqslant 1}
\end{aligned}
$$

слабо компактны.

Постоянные $A_{n}$ можно выбрать по формуле

$$
A_{n}=C_{n}+\operatorname{med}\left(\sum_{k=1}^{N_{n}}\left(m_{n, k}+\beta_{n, k}\right)\right), \quad n=1,2, \ldots
$$

где $C_{n}$ - любые вещественные числа, удовлетворяющие условию $\sup _{n}\left|C_{n}\right|<\infty$.

3 а м е ч а н и е 2. Теоремы 1 и 2 останутся справедливыми также в том случае, когда центрирующие постоянные $A_{n}$ равны нулю, если участвующие в формулировках теорем величины

$$
\sum_{k=1}^{N_{n}} \alpha_{n, k}-\operatorname{med}\left(\sum_{k=1}^{N_{n}} \alpha_{n, k}\right) \quad \text { и } \sum_{k=1}^{N_{n}}\left(m_{n, k}+\beta_{n, k}\right)-\operatorname{med}\left(\sum_{k=1}^{N_{n}}\left(m_{n, k}+\beta_{n, k}\right)\right)
$$

заменить на величины

$$
\sum_{k=1}^{N_{n}} \alpha_{n, k} \quad \text { и } \sum_{k=1}^{N_{n}}\left(m_{n, k}+\beta_{n, k}\right) .
$$

Теорема 3. Предположим, что выполняется условие (2). Для того чтобы при некотором подборе вещественных иисел $A_{n}$ последовательность случайных сумм $\left\{S_{N_{n}}^{(n)}-A_{n}\right\}_{n \geqslant 1}$ была слабо компактной, необходимо и достаточно, чтобъ

$$
\lim _{x \rightarrow \infty} \sup _{n} \mathbf{P}\left\{\sum_{k=1}^{N_{n}} \mathbf{P}\left\{\left|X_{n}, k\right| \geqslant x\right\} \geqslant \varepsilon\right\}=0
$$

для любого $\varepsilon>0$,

В) последовательности случайных величин

$$
\left\{\sum_{k=1}^{N_{n}} \mathbf{E}\left(\frac{\left.X_{n, k}-\alpha_{n, k}\right)^{2}}{1+\left(X_{n, k}-\alpha_{n, k}\right)^{2}}\right)\right\}_{n \geqslant 1}, \quad\left\{\sum_{k=1}^{N_{n}} \alpha_{n, k}-\operatorname{med}\left(\sum_{k=1}^{N_{n}} \alpha_{n, k}\right)\right\}_{n \geqslant 1}
$$

слабо компактны, где $\alpha_{n, k}=\alpha_{n, k}(v)$ определены перед теоремой 1 .

Постоянные $A_{n}$ можно выбрать по формуле, указанной в теоремe 1. 
Предположим дополнительно, что для каждого $n=1,2, \ldots$ случайные величины $X_{n, k}, k=1,2, \ldots$, одинаково распределены. Обозначим

$$
\begin{gathered}
\alpha_{n}=\alpha_{n}(v)=\int_{|x|<v} x d \mathbf{P}\left\{X_{n, 1}<x\right\} \\
d_{n}=d_{n}(v)=\mathbf{E}\left(\frac{\left(X_{n, 1}-\alpha_{n}\right)^{2}}{1+\left(X_{n, 1}-\alpha_{n}\right)^{2}}\right),
\end{gathered}
$$

где $v$ - произвольное положительное число.

Теорема 4. Предположим, что $N_{n} \rightarrow \infty$ по вероятности при $n \rightarrow$ $\infty$ и для каждого $n=1,2, \ldots$ случайные величины $X_{n, k}, k=1,2, \ldots$, одинаково распределены. Для того чтобы последовательность случайных сумм $\left\{S_{N_{n}}^{(n)}\right\}_{n \geqslant 1}$ была слабо компактной, необходимо и достаточно, чтобы выполнялось одно из следующих двух усіловий:

А) для почти всех по мере Лебеаа $q \in(0,1)$ последовательность случайных величин $\left\{S_{l_{n}(q)}^{(n)}\right\}_{n \geqslant 1}$ слабо компактна,

В) для почти всех по мере Лебеаа $q \in(0,1)$ последовательность случайных величин $\left\{\sum_{k=1}^{l_{n}(q)}\left(X_{n, k}-\alpha_{n}\right)\right\}_{n \geqslant 1}$ слабо компактна и числовая последовательность $\left\{l_{n}(q)\left|\alpha_{n}\right|\right\}_{n \geqslant 1}$ ограничена.

Обозначим $\delta_{n}=L\left(R_{n}, \mathscr{E}\right)$ расстояние Леви (см. $[1, \S 9$, теорема 1$\left.]\right)$ между функцией распределения $R_{n}(x)=\mathbf{P}\left\{N_{n}\left(d_{n}+\left|\alpha_{n}\right|\right)<x\right\}$, и функцией распределения $\mathscr{E}$ случайной величины, тождественно равной нулю,

$$
k_{n}= \begin{cases}l_{n}\left(1-\frac{1}{n+1}\right), & \text { если } d_{n}+\left|\alpha_{n}\right|=0, \\ {\left[\frac{1}{\left|\alpha_{n}\right|}\right],} & \text { если } d_{n}=0 \text { и }\left|\alpha_{n}\right|>0, \\ {\left[\frac{2 \delta_{n}}{d_{n}+\left|\alpha_{n}\right|}\right]+1,} & \text { если } d_{n}>0,\end{cases}
$$

где квадратные скобки обозначают целую часть числа, заключенного в них, $\alpha_{n}=\alpha_{n}(v)$ и $d_{n}=d_{n}(v)$ определены перед формулировкой теоремы 4.

Теорема 5. Предположим, что $N_{n} \rightarrow \infty$ по вероятности при $n \rightarrow$ ж и для каждого $n=1,2, \ldots$ случайные величины $X_{n, k}, k=1,2, \ldots$, одинаково распределены. Для того чтобы последовательность случайных сумм $\left\{S_{N_{n}}^{(n)}\right\}_{n \geqslant 1}$ была слабо компактной, необходимо и достаточно, чтобы последовательности случайных величин $\left\{N_{n} / k_{n}\right\}_{n \geqslant 1} u$ $\left\{S_{k_{n}}^{(n)}\right\}_{n \geqslant 1}$ были слабо компактными, где числа $k_{n}$ определены в (3).

Обратимся к задаче, решенной в [5]. Следуя автору статьи [5], назовем последовательность случайных величин $\left\{X_{n}\right\}_{n \geqslant 1}$ стохастически отделимой от нуля, если из нее нельзя выделить подпоследовательность, сходящуюся по вероятности к нулю. 
3 а м е ч а н и е 3 . Определим $\alpha_{n}=\alpha_{n}(v)$ и $d_{n}=d_{n}(v)$, как указано перед формулировкой теоремы теоремы 4 , и предположим, что $d_{n}>0$ для всех $n$. Для того чтобы стохастически отделимая от нуля последовательность случайных сумм $\left\{S_{N_{n}}^{(n)}\right\}_{n \geqslant 1}$ была слабо компактной, необходимо и достаточно, чтобы последовательности случайных величин $\left\{N_{n} / k_{n}\right\}_{n \geqslant 1}$ и $\left\{S_{k_{n}}^{(n)}\right\}_{n \geqslant 1}$ были слабокомпактными, где $k_{n}=\left[1 /\left(d_{n}+\right.\right.$ $\left.\left.\left|\alpha_{n}\right|\right)\right]$ - целая часть числа $1 /\left(d_{n}+\left|\alpha_{n}\right|\right)$.

Это утверждение представляет собой некоторую конкретизацию теоремы Д. Сааса (см. доказательство теоремы 1 в [5]), в которой числа $k_{n}$ не строятся, как в замечании 3 , а утверждается существование последовательности натуральных чисел $\left\{k_{n}\right\}_{n \geqslant 1}$, для которой последовательности $\left\{N_{n} / k_{n}\right\}_{n \geqslant 1}$ и $\left\{S_{k_{n}}^{(n)}\right\}_{n \geqslant 1}$ слабо компактны.

Естественно поставить вопрос об аналоге теоремы 5 в отношении пентрированных сумм. Ответ на этот вопрос содержится в следуюшей теореме, доказательство которой, как будет показано, состоит в комбинации теорем 2 и 5 . Обозначим $m_{n}$ медиану случайной величины $X_{n, 1}$,

$$
\begin{aligned}
\beta_{n} & =\int_{|x|<v} x d \mathbf{P}\left\{X_{n, 1}-m_{n}<x\right\}, \\
\gamma_{n} & =\int_{|x|<v} x d \mathbf{P}\left\{X_{n, 1}-m_{n}-\beta_{n}<x\right\}, \\
\Delta_{n} & =\Delta_{n}(v)=\mathbf{E}\left(\frac{\left(X_{n, 1}-m_{n}-\beta_{n}-\gamma_{n}\right)^{2}}{1+\left(X_{n, 1}-m_{n}-\beta_{n}-\gamma_{n}\right)^{2}}\right), \\
k_{n} & = \begin{cases}l_{n}\left(1-\frac{1}{n+1}\right), & \text { если } \Delta_{n}+\left|\gamma_{n}\right|=0, \\
{\left[\frac{1}{\left|\gamma_{n}\right|}\right],} & \text { если } \Delta_{n}=0 \text { и }\left|\gamma_{n}\right|>0, \\
{\left[\frac{2 \varepsilon_{n}}{\Delta_{n}+\left|\gamma_{n}\right|}\right]+1,} & \text { если } \Delta_{n}>0,\end{cases}
\end{aligned}
$$

где $\varepsilon_{n}$ обозначает расстояние Леви между функциями распределения $\mathscr{E}(x)$ и $\mathbf{P}\left\{N_{n}\left(\Delta_{n}+\left|\gamma_{n}\right|\right)<x\right\}, v-$ произвольное положительное число.

Теорема 6. Предположим, что $N_{n} \rightarrow \infty$ по вероятности при $n \rightarrow \infty$ и для каждого $n=1,2, \ldots$ случайные величины $X_{n, k}, k=$ $1,2, \ldots$, одинаково распределены. Для того чтобы при некотором подборе вещественных чисел $A_{n}$ последовательность случайньх сумм $\left\{S_{N_{n}}^{(n)}-A_{n}\right\}_{n \geqslant 1}$ была слабо компактной, необходимо и достаточно, чтобы последовательности случайных величин $\left\{N_{n} / k_{n}\right\}_{n \geqslant 1},\left\{N_{n}\left(m_{n}+\right.\right.$ $\left.\left.\beta_{n}\right)-\operatorname{med}\left(N_{n}\left(m_{n}+\beta_{n}\right)\right)\right\}_{n \geqslant 1} u\left\{S_{k_{n}}^{(n)}-k_{n}\left(m_{n}+\beta_{n}\right)\right\}_{n \geqslant 1}$ были слабо компактными, где числа $k_{n}$ определены 6 (4).

Постоянные $A_{n}$ можно выбрать по формуле

$$
A_{n}=C_{n}+\operatorname{med}\left(N_{n}\left(m_{n}+\beta_{n}\right)\right), \quad n=1,2, \ldots,
$$


где $C_{n}$ - любые вещественные числа, удовлетворяющие условию $\sup _{n}\left|C_{n}\right|<\infty$.

3 а м е ч а н и е 4 . Теорема 6 останется справедливой также в том случае, когда пентрируюшие постоянные $A_{n}$ равны нулю, если условие слабой компактности последовательности $\left\{N_{n}\left(m_{n}+\beta_{n}\right)-\operatorname{med}\left(N_{n}\left(m_{n}+\right.\right.\right.$ $\left.\left.\left.\beta_{n}\right)\right)\right\}_{n \geqslant 1}$ заменить на условие слабой компактности последовательности $\left\{N_{n}\left(m_{n}+\beta_{n}\right)\right\}_{n \geqslant 1}$.

Доказательства теорем будут основаны на вспомогательных утверждениях, которые приведены в следующем разделе.

\section{2. Леммы}

Нам понадобится понятие безгранично делимой функции распределения, которое мы считаем известным. В соответствии с классическим результатом $[1, \S 18$, теорема 1$]$ функция распределения $F$ является безгранично делимой тогда и только тогда, когда ее характеристическая функция $f$ допускает следуюшее представление, называемое представлением Леви-Хинчина,

$$
f(t)=\exp \left\{i \gamma t+\int_{-\infty}^{\infty}\left(e^{i t x}-1-\frac{i t x}{1+x^{2}}\right) \frac{1+x^{2}}{x^{2}} d G(x)\right\}
$$

где $i=\sqrt{-1}-$ мнимая единица, $\gamma$ - вешественное число, а $G$-- неубываюцая, непрерывная слева функция, причем $G(-\infty)=0$ и $G(\infty)<\infty$.

Лемма 1. Пусть дана последовательность серий $X_{n, k}, 1 \leqslant k \leqslant k_{n}$, $n=1,2, \ldots$, независиных в каждой серии случайных величин, удовлетворяющих условию

$$
\max _{1 \leqslant k \leqslant k_{n}} \mathbf{P}\left\{\left|X_{n, k}\right| \geqslant \varepsilon\right\} \longrightarrow 0, \quad \forall \varepsilon>0 \text { npu } n \rightarrow \infty
$$

Пусть характеристическая функчия $f$ безгранично делимой функчии распределения $F$ имеет представление (5). Для того чтобы при некотором подборе вещественных постоянных $A_{n}$ выполнялось соотночение

$$
\mathbf{P}\left\{\sum_{k=1}^{k_{n}} X_{n, k}-A_{n}<x\right\} \Longrightarrow F(x) \quad \text { npu } n \rightarrow \infty
$$

необходимо и достаточно, чтобы

1) в точках непрерьвности $x, x \neq 0$, функции $G$

$$
\begin{aligned}
& \lim _{n \rightarrow \infty} \sum_{k=1}^{k_{n}} \mathbf{P}\left\{X_{n, k}<x\right\}=\int_{-\infty}^{x} \frac{1+y^{2}}{y^{2}} d G(y), \quad x<0, \\
& \lim _{n \rightarrow \infty} \sum_{k=1}^{k_{n}} \mathbf{P}\left\{X_{n, k} \geqslant x\right\}=\int_{x}^{\infty} \frac{1+y^{2}}{y^{2}} d G(y), \quad x>0,
\end{aligned}
$$




$$
\begin{gathered}
\text { 2) } \lim _{\varepsilon \rightarrow 0} \limsup _{n \rightarrow \infty} \mathscr{D}_{n}(\varepsilon)=G(0+)-G(0)=\lim _{\varepsilon \rightarrow 0} \liminf _{n \rightarrow \infty} \mathscr{D}_{n}(\varepsilon) \\
\mathscr{D}_{n}(\varepsilon)=\sum_{k=1}^{k_{n}}\left\{\int_{|x|<\varepsilon} x^{2} d \mathbf{P}\left\{X_{n, k}<x\right\}-\left(\int_{|x|<\varepsilon} x d \mathbf{P}\left\{X_{n, k}<x\right\}\right)^{2}\right\} .
\end{gathered}
$$

Постоянные $A_{n}$ можно выбрать по формуле

$$
A_{n}=\sum_{k=1}^{k_{n}} \int_{|x|<\tau} x d \mathbf{P}\left\{X_{n, k}<x\right\}-\gamma(\tau),
$$

zдe

$$
\gamma(\tau)=\gamma+\int_{|x|<\tau} x d G(x)-\int_{|x| \geqslant \tau} \frac{1}{x} d G(x),
$$

а число $\tau>0$ выбрано так, что $\pm \tau$ являются точками непрерывности функиии $G$.

Д о к а з а т е л в с т в о. См. $[1, \S 25$, теорема 4].

Следующая лемма представляет собой критерий сдвиг-компактности последовательности сумм независимых случайных величин, удовлетворяюших условию равномерной малости. Едва ли эта лемма является новой, но нам не удалось найти источник, на который можно было бы сослаться.

Лемма 2. Пусть дана последовательность серий $X_{n, k}, 1 \leqslant k \leqslant k_{n}$, $n=1,2, \ldots$, независимых в каждой серии случайных величин, удовлетворяющих условию (6). Для того чтобы при некотором подборе вещественньх чисел $A_{n}$ последовательность случайных величин $\left\{S_{k_{n}}\right.$ $\left.A_{n}\right\}_{n \geqslant 1}, S_{k_{n}}=X_{n, 1}+\cdots+X_{n, k_{n}}$, была слабо компактной, необходимо и достаточно, чтобы

$$
\begin{gathered}
\lim _{x \rightarrow \infty} \sup _{n} \sum_{k=1}^{k_{n}} \mathbf{P}\left\{\left|X_{n, k}\right| \geqslant x\right\}=0, \\
\sup _{n} \sum_{k=1}^{k_{n}} \mathbf{E}\left(\frac{\left.X_{n, k}-\alpha_{n, k}\right)^{2}}{1+\left(X_{n, k}-\alpha_{n, k}\right)^{2}}\right)<\infty .
\end{gathered}
$$

Постоянные $A_{n}$ можно выбрать по формуле

$$
A_{n}=\sum_{k=1}^{k_{n}} \alpha_{n, k}, \quad n=1,2, \ldots
$$

где $\alpha_{n, k}=\alpha_{n, k}(v)$ определень перед формулировкой теоремы 1 .

Д ок азатель с т в о. Н е обходи м о ть. Предположим, что (7) не имеет места. В этом случае будет выполняться неравенство

$$
\sum_{k=1}^{k_{n}} \mathbf{P}\left\{\left|X_{n, k}\right| \geqslant x_{n}\right\} \geqslant \alpha
$$


для некоторого числа $\alpha>0$ и некоторой числовой последовательности $\left\{x_{n}\right\}, n \in \mathcal{N}$, сходящейся к бесконечности. Выберем подпоследовательность функций распределения $\left\{F_{n}\right\}, n \in \mathscr{N}_{1} \subset \mathcal{N}, F_{n}(x)=\mathbf{P}\left\{S_{k_{n}}-A_{n}<\right.$ $x\}$, которая слабо сходится к некоторой функции распределения $\boldsymbol{F}$. По теореме Хинчина $[1, \S 24$, теорема 2] функция распределения $F$ безгранично делима. $\mathrm{K}$ последовательности $\left\{S_{k_{n}}-A_{n}\right\}, n \in \mathcal{N}_{1}$, применима. лемма 1, по которой

$$
\lim _{x \rightarrow \infty} \sup _{n \in \mathscr{N}_{1}} \sum_{k=1}^{k_{n}} \mathbf{P}\left\{\left|X_{n, k}\right| \geqslant x\right\}=0
$$

в противоречие нашему предположению. Условие (7) показано. Обозначим $J_{n}$ сумму величин, стояших в (8) под знаком точной верхней грани. Утверждение (8) представляет собой незначительное обобщение известного утверждения $[1, \S 23$, теорема 2$]$, согласно которому оно выполняется, если последовательность $\left\{S_{k_{n}}-A_{n}\right\}_{n \geqslant 1}$ слабо сходится. Действительно, предположим, что (8) не имеет места. В этом случае найдется последовательность натуральных чисел $\mathcal{N}$, что $J_{n} \rightarrow \infty$ при $n \rightarrow \infty$, $n \in \mathcal{N}$. Выберем подпоследовательность $\mathcal{N}_{1} \subset \mathcal{N}$ таким образом, чтобы последовательность случайных величин $\left\{S_{k_{n}}-A_{n}\right\}, n \in \mathcal{N}_{1}$, слабо сходилась. По упомянутому утверждению из монографии [1] мы имеем, что $\sup \left\{J_{n}: n \in \mathcal{N}_{1}\right\}<\infty$, в противоречие предположению. Необходимость доказана.

Д ос т а т ч н о с т ь. Из условия (6) следует, что

$$
\lim _{n \rightarrow \infty} \max _{1 \leqslant k \leqslant k_{n}}\left|\alpha_{n, k}\right|=0
$$

Поэтому наряду с (7) выполняется следуюшее соотношение

$$
\lim _{x \rightarrow \infty} \sup _{n} \sum_{k=1}^{k_{n}} \mathbf{P}\left\{\left|X_{n, k}-\alpha_{n, k}\right| \geqslant x\right\}=0
$$

и, следовательно,

$$
\lim _{x \rightarrow \infty} \sup _{n} \sum_{k=1}^{k_{n}} \int_{|u| \geqslant x} \frac{u^{2}}{1+u^{2}} d \mathbf{P}\left\{X_{n, k}-\alpha_{n, k}<u\right\}=0 .
$$

Это соотношение и условие (8) являются необходимыми и достаточными условиями для вполне компактности последовательность функций $\left\{G_{n}\right\}_{n \geqslant 1}$

$$
G_{n}(x)=\sum_{k=1}^{k_{n}} \int_{-\infty}^{x} \frac{u^{2}}{1+u^{2}} d \mathbf{P}\left\{X_{n, k}-\alpha_{n, k}<u\right\} .
$$

Другими словами (см. [3, с. 191]), какова бы ни была последовательность натуральных чисел $\mathscr{N}$, найдется подпоследовательность $\mathscr{N}_{1} \subset \mathcal{N}$, 
такая, что последовательность функций $\left\{G_{n}\right\}, n \in \mathscr{N}_{1}$, слабо сходится к некоторой функции $G$ и $G_{n}( \pm \infty) \rightarrow G( \pm \infty)$ при $n \rightarrow \infty, n \in \mathscr{N}_{1}$. В соответствии с известным утверждением (см. $[1, \S 25$, с. 128 , примечание]) найдется безгранично делимая функция распределения $F$, такая, что

$$
\mathbf{P}\left\{S_{k_{n}}-B_{n}<x\right\} \Longrightarrow F(x)
$$

при $n \rightarrow \infty, n \in \mathscr{N}_{1}$, где $B_{n}$ - некоторые вещественные числа. Характеристическую функцию, соответствуюшую функции распределения $F$, можно записать в виде (5). По лемме 1 в качестве чисел $B_{n}$ можно взять

$$
B_{n}=\sum_{k=1}^{k_{n}} \int_{|x|<\tau} x d \mathbf{P}\left\{X_{n, k}<x\right\}, \quad n \in \mathscr{N}_{1} .
$$

Пусть величина $A_{n}$ определена, как указано в формулировке леммы. Из (9) следует, что последовательность $\left\{S_{k_{n}}-A_{n}\right\}, n \in \mathscr{N}_{1}$, слабо компактна, если числовая последовательность $\left\{\left|D_{n}\right|\right\}, n \in \mathscr{N}_{1}$, ограничена, где

$$
D_{n}=\sum_{k=1}^{k_{n}}\left(\int_{|x|<\tau} x d \mathbf{P}\left\{X_{n, k}<x\right\}-\int_{|x|<v} x d \mathbf{P}\left\{X_{n, k}<x\right\}\right) .
$$

Величина $\left|D_{n}\right|$ удовлетворяет неравенству

$$
\left|D_{n}\right| \leqslant d \sum_{k=1}^{k_{n}}\left(\mathbf{P}\left\{c \leqslant X_{n, k} \leqslant d\right\}+\mathbf{P}\left\{-d \leqslant X_{n, k} \leqslant-c\right\}\right),
$$

где $c=\min \{v, \tau\}, d=\max \{v, \tau\}$. Из этого неравенства и условия 1) леммы 1 , которое выполняется при $n \rightarrow \infty, n \in \mathscr{N}_{1}$, следует, что последовательность $\left\{\left|D_{n}\right|\right\}, n \in \mathscr{N}_{1}$, ограничена. Таким образом доказано, что из любой последовательности $\left\{S_{k_{n}}-A_{n}\right\}, n \in \mathcal{N}$, можно выделить подпоследовательность, которая слабо сходится. Лемма доказана.

Лемма 3. Пусть дана последовательность серий $X_{n, k}, 1 \leqslant k \leqslant k_{n}$, $n=1,2, \ldots$, независимьх в каждой серии случайных величин, удовлетворяющих условию (6). Если

$$
\lim _{n \rightarrow \infty} \sum_{k=1}^{k_{n}} \mathbf{E}\left(\frac{\left(X_{n, k}-\alpha_{n, k}\right)^{2}}{1+\left(X_{n, k}-\alpha_{n, k}\right)^{2}}\right)=0,
$$

где величины $\alpha_{n, k}=\alpha_{n, k}(v)$ определены перед формулировкой теоремbl 1, mo

$$
\sum_{k=1}^{k_{n}}\left(X_{n, k}-\alpha_{n, k}\right) \stackrel{\mathbf{p}}{\longrightarrow} 0 \text { npu } n \longrightarrow \infty .
$$


Д о к а 3 а т е лг ь с т в о. Эта лемма представляет собой непосредственное следствие леммы 1. Действительно, из условия леммы и леммы 1 следует (см. $[1, \S 25$, с. 128 , примечание]), что

$$
\sum_{k=1}^{k_{n}} X_{n, k}-A_{n} \stackrel{\mathrm{p}}{\rightarrow} 0 \quad \text { при } n \rightarrow \infty
$$

при некотором выборе постоянных $A_{n}$. Характеристическая функция случайной величины, равной нулю, имеет представление (5), где $\gamma=0$ и $G(x)=0$ для всех $x$. Точка $x=v>0$ является точкой непрерывности функции $G$ и, следовательно, по лемме 1 центрирующие постоянные $A_{n}$ можно выбрать, как указано в лемме 2. Лемма доказана.

Назовем левой (соответственно правой) $q$-квантилью, $q \in(0,1)$, случайной величины $X$ (функции распределения $F(x)=\mathbf{P}\{X<x\}$ ) число

$$
l(q)=\inf \{x: F(x) \geqslant q\}(\text { соответственно } r(q)=\sup \{x: F(x) \leqslant q\}) .
$$

Лемма 4. Пусть даны функчии распределения $F, F_{n}, n=1,2, \ldots$. Обозначим $l(q)$ и $r(q)$ (соответственно $l_{n}(q)$ и $r_{n}(q)$ ) левые и правые q-квантили функчии распределения $F$ (соответственно $F_{n}$ ). Последовательность $\left\{F_{n}\right\}_{n \geqslant 1}$ слабо сходится $\kappa F$ тогда и только тогда, когда

$$
l(q) \leqslant \liminf _{n \rightarrow \infty} l_{n}(q) \leqslant \limsup _{n \rightarrow \infty} r_{n}(q) \leqslant r(q)
$$

для всех $q \in(0,1)$. Более того, $l(q)=r(q)$ почти всюду по мере Лебега.

Д о к а 3 а т е л с с т о. См. [2, теорема 1.1.1] или [9].

Лемма 5. Пусть даны случайные величины $X$ и $\lambda$, причем $\lambda$ равномерно распределена на $(0,1)$. Обозначим $l(q)$ левую q-квантиль $X$. Случайные величины $X$ и $l(\lambda)$ одинаково распределены.

Д о к а з а т е л с т в о. Утверждение непосредственно следует из определения понятия левой $q$-квантили.

Лемма 6. Пусть даны последовательности случайных величин $\left\{X_{n}\right\}_{n \geqslant 1},\left\{Y_{n}\right\}_{n \geqslant 1},\left\{Z_{n}\right\}_{n \geqslant 1}$. Iредположим, что $Z_{n}=X_{n}+Y_{n}, n=$ $1,2, \ldots$. Если пюбые две последовательности из этих трех слабо компактны, тогда третья последовательность также слабо компактна.

Д ок а за т е л с т в о. Утверждение следует из критерия (1).

\section{3. Доказательства теорем}

Доказательство те оремы 1. Н еобходи мость. Докажем сначала, что условие В) является следствием условия А). Для каждого $n=1,2, \ldots$ построим независимые случайные величины $\lambda, N_{n}^{\prime}$, $X_{n, k}^{\prime}, k=1,2, \ldots$, на некотором вероятностном пространстве $\left(\Omega^{\prime}, \mathscr{A}^{\prime}, \mathbf{P}^{\prime}\right)$ таким образом, чтобы $\lambda$ была равномерно распределена на $(0,1), N_{n}^{\prime} \stackrel{d}{=}$ 
$N_{n}, X_{n, k}^{\prime} \stackrel{\mathrm{d}}{=} X_{n, k}$. Такие случайные величины можно построить по известной теореме Колмогорова $[3$, с. 103]. Без ограничения общности можно считать, что вероятностные пространства $\left(\Omega^{\prime}, \mathscr{A}^{\prime}, \mathbf{P}^{\prime}\right)$ и $(\Omega, \mathscr{A}, \mathbf{P})$ совпадают и $N_{n}^{\prime}=N_{n}, X_{n, k}^{\prime}=X_{n, k}$. По лемме 5 случайные величины $N_{n}$ и $l_{n}(\lambda)$ одинаково распределены и, следовательно, этим свойством также обладают случайные величины $V_{n}$ и $V_{n}(\lambda)$, где

$$
V_{n}=\sum_{k=1}^{N_{n}}\left(X_{n, k}-\alpha_{n, k}\right), \quad V_{n}(q)=\sum_{k=1}^{l_{n}(q)}\left(X_{n, k}-\alpha_{n, k}\right), \quad q \in(0,1) .
$$

Из равенств

$$
\mathbf{P}\left\{\left|V_{n}\right| \geqslant x\right\}=\mathbf{P}\left\{\left|V_{n}(\lambda)\right| \geqslant x\right\}=\int_{0}^{1} \mathbf{P}\left\{\left|V_{n}(q)\right| \geqslant x\right\} d q
$$

следует, что

$$
\sup _{n} \mathbf{P}\left\{\left|V_{n}\right| \geqslant x\right\} \leqslant \int_{0}^{1} \sup _{n} \mathbf{P}\left\{\left|V_{n}(q)\right| \geqslant x\right\} d q \longrightarrow 0
$$

при $x \rightarrow \infty$, так как для почти всех по мере Лебега $q \in(0,1)$

$$
\lim _{x \rightarrow \infty} \sup _{n} \mathbf{P}\left\{\left|V_{n}(q)\right| \geqslant x\right\}=0
$$

по критерию (1) в силу условия А). По критерию (1) последовательность случайных величин $\left\{V_{n}\right\}_{n \geqslant 1}$ слабо компактна.

Докажем, что выполнено условие А). Для каждого $n=1,2, \ldots$ построим независимые случайные величины $N_{n}^{\prime}, X_{n, k}^{\prime}, X_{n, k}^{\prime \prime}, k=1,2, \ldots$, на некотором вероятностном пространстве $\left(\Omega^{\prime}, \mathscr{A}^{\prime}, \mathbf{P}^{\prime}\right)$ таким образом, чтобы выполнялись следующие соотношения $N_{n}^{\prime} \stackrel{\mathrm{d}}{=} N_{n}, X_{n, k}^{\prime} \stackrel{\mathrm{d}}{=} X_{n, k}$, $X_{n, k}^{\prime \prime} \stackrel{\mathrm{d}}{=} X_{n, k}$. Без ограничения обшности можно считать, что вероятностные пространства $\left(\Omega^{\prime}, \mathscr{A}^{\prime}, \mathbf{P}^{\prime}\right)$ и $(\Omega, \mathscr{A}, \mathbf{P})$ совпадают и $N_{n}^{\prime}=N_{n}$, $X_{n, k}^{\prime \prime}=X_{n, k}$. Заметим, что случайные величины $Z_{n}=S_{N_{n}}^{(n)}-A_{n}$ и $Z_{n}^{\prime}=\sum_{k=1}^{N_{n}} X_{n, k}^{\prime}-A_{n}$ одинаково распределены. По формуле полной вероятности мы имеем

$$
\begin{aligned}
\mathbf{P}\left\{\left|Z_{n}-Z_{n}^{\prime}\right| \geqslant x\right\} & =\sum_{r=1}^{\infty} \mathbf{P}\left\{N_{n}=r\right\} \mathbf{P}\left\{\left|\sum_{k=1}^{r}\left(X_{n, k}-X_{n, k}^{\prime}\right)\right| \geqslant x\right\} \\
& \geqslant \sum_{r=l_{n}(q)}^{\infty} \mathbf{P}\left\{N_{n}=r\right\} \mathbf{P}\left\{\left|\sum_{k=1}^{r}\left(X_{n, k}-X_{n, k}^{\prime}\right)\right| \geqslant x\right\} .
\end{aligned}
$$

Для упрощения последующих записей обозначим

$$
W_{n}(q)=S_{l_{n}(q)}^{(n)}=\sum_{k=1}^{l_{n}(q)} X_{n, k}, \quad W_{n}^{\prime}(q)=\sum_{k=1}^{l_{n}(q)} X_{n, k}^{\prime} .
$$


По неравенству Леви [3, с. 261] для симметричных случайных величин мы имеем

$$
\mathbf{P}\left\{\left|W_{n}(q)-W_{n}^{\prime}(q)\right| \geqslant x\right\} \leqslant 2 \mathbf{P}\left\{\left|\sum_{k=1}^{r}\left(X_{n, k}-X_{n, k}^{\prime}\right)\right| \geqslant x\right\}
$$

для всех $r=l_{n}(q), l_{n}(q)+1, \ldots$ Поэтому

$$
\frac{1-q}{2} \mathbf{P}\left\{\left|W_{n}(q)-W_{n}^{\prime}(q)\right| \geqslant x\right\} \leqslant \mathbf{P}\left\{\left|Z_{n}-Z_{n}^{\prime}\right| \geqslant x\right\}
$$

и, следовательно,

$$
\mathbf{P}\left\{\left|W_{n}(q)-W_{n}^{\prime}(q)\right| \geqslant x\right\} \leqslant \frac{4}{1-q} \mathbf{P}\left\{\left|Z_{n}\right| \geqslant \frac{x}{2}\right\}
$$

так как $\mathbf{P}\left\{\left|Z_{n}-Z_{n}^{\prime}\right| \geqslant x\right\} \leqslant 2 \mathbf{P}\left\{\left|Z_{n}\right| \geqslant x / 2\right\}$. В силу неравенства симметризации $[3$, с. 259]

$$
\mathbf{P}\left\{\left|W_{n}(q)-\operatorname{med} W_{n}(q)\right| \geqslant x\right\} \leqslant 2 \mathbf{P}\left\{\left|W_{n}(q)-W_{n}^{\prime}(q)\right| \geqslant x\right\}
$$

и (11) мы имеем, что

$$
\mathbf{P}\left\{\left|W_{n}(q)-\operatorname{med} W_{n}(q)\right| \geqslant x\right\} \leqslant \frac{8}{1-q} \mathbf{P}\left\{\left|Z_{n}\right| \geqslant \frac{x}{2}\right\}
$$

По критерию (1) из (12) следует, что последовательность случайных величин $\left\{W_{n}(q)-\operatorname{med} W_{n}(q)\right\}_{n \geqslant 1}, q \in(0,1)$, слабо компактна. Докажем, что последовательность $\left\{V_{n}(q)\right\}_{n \geqslant 1}, V_{n}(q)=W_{n}(q)-\sum_{k=1}^{l_{n}(q)} \alpha_{n, k}$, также слабо компактна. Действительно, можно доказать (см. [2, с. 43]), что условие (2) равносильно условию

$$
\lim _{n \rightarrow \infty} \max _{1 \leqslant k \leqslant l_{n}(q)} \mathbf{P}\left\{\left|X_{n, k}\right| \geqslant \varepsilon\right\}=0, \quad \forall \varepsilon>0 \text { и } q \in(0,1) .
$$

По лемме 2 вместо центрируюших постоянных $\operatorname{med} W_{n}(q)$ можно взять величины $\alpha_{n, 1}+\alpha_{n, 2}+\cdots+\alpha_{n, l_{n}(q)}$.

Выше было доказано, что слабая компактность последовательности случайных величин $\left\{V_{n}(q)\right\}_{n \geqslant 1}$ для почти всех по мере Лебега $q \in(0,1)$ влечет слабую компактность последовательности $\left\{V_{n}\right\}_{n \geqslant 1}$. Из равенства

$$
S_{N_{n}}^{(n)}-A_{n}=V_{n}+\sum_{k=1}^{N_{n}} \alpha_{n, k}-A_{n}, \quad n=1,2, \ldots
$$


и неравенства симметризации [3, с. 259]

$$
\begin{array}{r}
\mathbf{P}\left\{\left|U_{n}\right| \geqslant x\right\} \leqslant 2 \mathbf{P}\left\{\left|\sum_{k=1}^{N_{n}} \alpha_{n, k}-A_{n}\right| \geqslant \frac{x}{2}\right\}, \\
U_{n}=\sum_{k=1}^{N_{n}} \alpha_{n, k}-\operatorname{med}\left(\sum_{k=1}^{N_{n}} \alpha_{n, k}\right),
\end{array}
$$

следует, что

$$
\mathbf{P}\left\{\left|U_{n}\right| \geqslant x\right\} \leqslant 2 \mathbf{P}\left\{\left|S_{N_{n}}^{(n)}-A_{n}\right| \geqslant \frac{x}{4}\right\}+2 \mathbf{P}\left\{\left|V_{n}\right| \geqslant \frac{x}{4}\right\}
$$

и, следовательно, последовательность случайных величин $\left\{U_{n}\right\}_{n \geqslant 1}$ слабо компактна. Необходимость доказана.

Д ос т а т ч н о с т. При доказательстве необходимости было установлено, что условие А) влечет условие В). Достаточность условия В) вытекает из равенства

$$
S_{N_{n}}^{(n)}-\operatorname{med}\left(\sum_{k=1}^{N_{n}} \alpha_{n, k}\right)=V_{n}+U_{n}, \quad n=1,2, \ldots,
$$

и леммы 6. Достаточность доказана.

Нам осталось оправдать законность выбора центрирующих постоянных по указанной формуле. Если при некотором выборе $A_{n}$ последовательность случайных сумм $\left\{S_{N_{n}}^{(n)}-A_{n}\right\}_{n \geqslant 1}$ слабо компактна, то из условия В) следует, что последовательность случайных сумм, стоящих слева в равенстве (13), также слабо компактна. Поэтому числовая последовательность $\left\{\left|C_{n}\right|\right\}_{n \geqslant 1}$,

$$
\begin{aligned}
& C_{n}=A_{n}-B_{n}=\left(S_{N_{n}}^{(n)}-B_{n}\right)-\left(S_{N_{n}}^{(n)}-A_{n}\right), \\
& B_{n}=\operatorname{med}\left(\sum_{k=1}^{N_{n}} \alpha_{n, k}\right), \quad n=1,2, \ldots,
\end{aligned}
$$

ограничена. Теорема доказана.

Доказатель с т в $о$ а меч ан ия 1. Замечание, фактически, было доказано попутно при доказательстве теоремы 1. Действительно, из равенства

$$
S_{l_{n}(q)}^{(n)}-B_{n}=V_{n}(q)+\sum_{k=1}^{l_{n}(q)} \alpha_{n, k}-B_{n}, \quad n=1,2, \ldots
$$

и леммы 6 следует, что последовательность случайных величин, стояших слева в (14), слабо компактна для почти всех по мере Лебега 
$q \in(0,1)$ и, следовательно, $\lim _{x \rightarrow \infty} \sup _{n} \mathbf{P}\left\{\left|S_{l_{n}(q)}^{(n)}-B_{n}\right| \geqslant x\right\}=0$. Из равенства

$$
\mathbf{P}\left\{\left|S_{N_{n}}^{(n)}-B_{n}\right| \geqslant x\right\}=\int_{0}^{1} \mathbf{P}\left\{\left|S_{l_{n}(q)}^{(n)}-B_{n}\right| \geqslant x\right\} d q
$$

которое можно доказать так же, как было доказано (10), следует, что

$$
\sup _{n} \mathbf{P}\left\{\left|S_{N_{n}}^{(n)}-B_{n}\right| \geqslant x\right\} \leqslant \int_{0}^{1} \sup _{n} \mathbf{P}\left\{\left|S_{l_{n}(q)}^{(n)}-B_{n}\right| \geqslant x\right\} d q \longrightarrow 0
$$

при $x \rightarrow \infty$. Замечание доказано.

Дока 3 а т л л с т о т е ре м 2 не приводится, так как оно почти буквально совпадает с доказательством теоремы 1.

Доказательст во за ме ч ан ия 2. Оно состоит в формальном повторении доказательств теорем 1 и 2 . Например, если последовательность случайных сумм $\left\{S_{N_{n}}^{(n)}\right\}_{n \geqslant 1}$ слабо компактна, то по теореме 1 последовательность $\left\{V_{n}\right\}_{n \geqslant 1}$ слабо компактна. Так как

$$
S_{N_{n}}^{(n)}=V_{n}+U_{n}^{\prime}, \quad U_{n}^{\prime}=\sum_{k=1}^{N_{n}} \alpha_{n, k}
$$

то по лемме 6 последовательность случайных величин $\left\{U_{n}^{\prime}\right\}_{n \geqslant 1}$ слабо компактна. Замечание доказано.

Доказательство те оремы 3. Н еобходимость. По условию В) теоремы 1 вторая из двух перечисленных последовательностей в условии В) слабо компактна. По условию А) теоремы 1 послецовательность $\left\{\sum_{k=1}^{l_{n}(q)}\left(X_{n, k}-\alpha_{n, k}\right)\right\}_{n \geqslant 1}$ слабо компактна для почти всех по мере Лебега $q \in(0,1)$. По лемме 2 для таких $q \in(0,1)$ выполняются следуюшие соотношения:

$$
\begin{gathered}
\lim _{x \rightarrow \infty} \sup _{n} \sum_{k=1}^{l_{n}(q)} \mathbf{P}\left\{\left|X_{n, k}\right| \geqslant x\right\}=0, \\
\sup _{n} \sum_{k=1}^{l_{n}(q)} \mathbf{E}\left(\frac{\left.X_{n, k}-\alpha_{n, k}\right)^{2}}{1+\left(X_{n, k}-\alpha_{n, k}\right)^{2}}\right)=C(q)<\infty .
\end{gathered}
$$

Докажем, что условие А) являются следствием (16). Для любых чисел $x>0$ и $\varepsilon>0$ выполняется равенство

$$
\mathbf{P}\left\{\sum_{k=1}^{N_{n}} \mathbf{P}\left\{\left|X_{n, k}\right| \geqslant x\right\} \geqslant \varepsilon\right\}=\int_{0}^{1} \mathbf{P}\left\{\sum_{k=1}^{l_{n}(q)} \mathbf{P}\left\{\left|X_{n, k}\right| \geqslant x\right\} \geqslant \varepsilon\right\} d q
$$


которое можно доказать так же, как было доказано (10). Из этого равенства и (16) следует, что

$$
\begin{aligned}
& \sup _{n} \mathbf{P}\left\{\sum_{k=1}^{N_{n}} \mathbf{P}\left\{\left|X_{n, k}\right| \geqslant x\right\} \geqslant \varepsilon\right\} \\
& \leqslant \int_{0}^{1} \mathbf{P}\left\{\sup _{n} \sum_{k=1}^{l_{n}(q)} \mathbf{P}\left\{\left|X_{n, k}\right| \geqslant x\right\} \geqslant \varepsilon\right\} d q \longrightarrow 0
\end{aligned}
$$

при $x \rightarrow \infty$. Аналогично, из равенства

$$
\begin{aligned}
& \mathbf{P}\left\{\sum_{k=1}^{N_{n}} \mathbf{E}\left(\frac{\left(X_{n, k}-\alpha_{n, k}\right)^{2}}{1+\left(X_{n, k}-\alpha_{n, k}\right)^{2}}\right) \geqslant x\right\} \\
& =\int_{0}^{\mathbb{1}} \mathbf{P}\left\{\sum_{k=1}^{l_{n}(q)} \mathbf{E}\left(\frac{\left(X_{n, k}-\alpha_{n, k}\right)^{2}}{1+\left(X_{n, k}-\alpha_{n, k}\right)^{2}}\right) \geqslant x\right\} d q
\end{aligned}
$$

и (17) следует, что первая из двух последовательностей в условии В) слабо компактна. Необходимость доказана.

Д о с т а т ч н о с т. Докажем, что цля почти всех по мере Лебега $q \in(0,1)$ выполняются соотношения (16) и (17). По формуле полной вероятности мы имеем:

$$
\begin{aligned}
\mathbf{P}\left\{\sum_{k=1}^{N_{n}} \mathbf{P}\left\{\left|X_{n, k}\right| \geqslant x\right\} \geqslant \varepsilon\right\} & =\sum_{r=1}^{\infty} \mathbf{P}\left\{N_{n}=r\right\} \mathbf{P}\left\{\sum_{k=1}^{r} \mathbf{P}\left\{\left|X_{n, k}\right| \geqslant x\right\} \geqslant \varepsilon\right\} \\
& \geqslant \sum_{r=l_{n}(q)}^{\infty} \mathbf{P}\left\{N_{n}=r\right\} \mathbf{P}\left\{\sum_{k=1}^{r} \mathbf{P}\left\{\left|X_{n, k}\right| \geqslant x\right\} \geqslant \varepsilon\right\} \\
& \geqslant(1-q) \mathbf{P}\left\{\sum_{k=1}^{l_{n}(q)} \mathbf{P}\left\{\left|X_{n, k}\right| \geqslant x\right\} \geqslant \varepsilon\right\} .
\end{aligned}
$$

ІІривлекая условие А), мы видим, что

$$
\lim _{x \rightarrow \infty} \sup _{n} \mathbf{P}\left\{\sum_{k=1}^{l_{n}(q)} \mathbf{P}\left\{\left|X_{n, k}\right| \geqslant x\right\} \geqslant \varepsilon\right\}=0
$$

откуда следует (16). Аналогично докажем (17). Условия (16) и (17) по лемме 2 влекут слабую компактность последовательности случайных величин $\left\{V_{n}(q)\right\}_{n \geqslant 1}$ для почти всех по мере Лебега $q \in(0,1)$. Привлекая условие В), мы видим, что выполнено условие А) теоремы 1. Теорема доказана. 
Доказательствотеоремы 4. Н еобходи мость. Докажем сначала, что

$$
\begin{aligned}
& \max _{1 \leqslant k \leqslant N_{n}} \mathbf{P}\left\{\left|X_{n, k}-m_{n}\right| \geqslant \varepsilon\right\} \\
& \quad=\mathbf{P}\left\{\left|X_{n, 1}-m_{n}\right| \geqslant \varepsilon\right\} \longrightarrow 0 \forall \varepsilon>0 \text { при } n \rightarrow \infty .
\end{aligned}
$$

Предположим противное. В этом случае будет выполняться неравенство $\mathbf{P}\left\{\left|X_{n, 1}-m_{n}\right| \geqslant \varepsilon\right\} \geqslant \gamma$ для некоторых $\varepsilon>0$ и $\gamma>0$ и всех $n$ из некоторой последовательности $\mathcal{N}$ натуральных чисел. По критерию (1) из неравенства (11), где в определении случайной величины $Z_{n}$ нужно положить $A_{n}=0$, следует, что последовательность функций распределения случайных величин $W_{n}(q)-W_{n}^{\prime}(q), q \in(0,1), n=1,2, \ldots$, слабо компактна. Поэтому некоторая ее подпоследовательность с индексами $n \in \mathscr{N}_{1} \subset \mathscr{N}$ слабо сходится к некоторой функции распределения $F$. Обозначим $f_{n}$ и $f$ характеристические функции случайной величины $X_{n, 1}$ и функции распределения $F$. По теореме непрерывности $[1, \S 13$, теорема 1] выполняется соотношение

$$
\left|f_{n}(t)\right|^{2 l_{n}(q)} \longrightarrow f(t) \quad \text { при } n \rightarrow \infty, n \in \mathscr{N}_{1},
$$

для каждого вещественного числа $t$. Заметим, что $l_{n}(q) \rightarrow \infty$, так как по предположению $N_{n} \stackrel{\text { p }}{\longrightarrow} \infty$. Отсюда следует (см. $[1, \S 14$, теорема 4]), что

$$
\mathbf{P}\left\{\left|X_{n, 1}-X_{n, 1}^{\prime}\right| \geqslant \varepsilon\right\} \longrightarrow 0 \quad \text { при } n \rightarrow \infty, n \in \mathscr{N}_{1}
$$

Это ведет к противоречию с нашим предположением, так как по неравенству симметризации $[3$, с. 259]

$$
\mathbf{P}\left\{\left|X_{n, 1}-m_{n}\right| \geqslant \varepsilon\right\} \leqslant 2 \mathbf{P}\left\{\left|X_{n, 1}-X_{n, 1}^{\prime}\right| \geqslant \varepsilon\right\} .
$$

Докажем условие А). По теореме 2 (см. замечание 2) последовательность случайных величин $\left\{N_{n}\left(m_{n}+\beta_{n}\right)\right\}_{n \geqslant 1}$ слабо компактна. Пусть $\tilde{l}_{n}(q)$ обозначает левую $q$-квантиль случайной величины $N_{n}\left|m_{n}+\beta_{n}\right|$. Из леммы 3 вытекает, что для любого $q \in(0,1)$ числовая последовательность $\left\{\tilde{l}_{n}(q)\right\}_{n \geqslant 1}$ ограничена. Действительно, пусть $\tilde{l}_{n}(q) \longrightarrow \infty$ при $n \rightarrow \infty, n \in \mathscr{N}$, для некоторого $q \in(0,1)$ и некоторой последовательности натуральных чисел $\mathcal{N}$. Выберем слабо сходяшуюся последовательность случайных величин $\left\{N_{n}\left(m_{n}+\beta_{n}\right)\right\}, n \in \mathscr{N}_{1} \subset \mathcal{N}$. По лемме 4 подпоследовательность $\left\{\tilde{l}_{n}(q)\right\}, n \in \mathcal{N}_{1}$, ограничена, что противоречит нашему предположению. Привлекая очевидное неравенство $l_{n}(q)\left|m_{n}+\beta_{n}\right| \leqslant \tilde{l}_{n}(q)$, мы видим, что для каждого $q \in(0,1)$ числовая последовательность $\left\{l_{n}(q)\left|m_{n}+\beta_{n}\right|\right\}_{n \geqslant 1}$ ограничена. По условию А) теоремы 2 для почти всех по мере Лебега $q \in(0,1)$ последовательность $\left\{\bar{V}_{n}(q)\right\}_{n \geqslant 1}$, где $\bar{V}_{n}(q)=\sum_{k=1}^{l_{n}(q)}\left(X_{n, k}-m_{n}-\beta_{n}\right)$, слабо компактна и, сле- 
довательно, последовательность $\left\{S_{l_{n}(q)}^{(n)}\right\}_{n \geqslant 1}$ также обладает этим свойством, так как

$$
S_{l_{n}(q)}^{(n)}=\bar{V}_{n}(q)+l_{n}(q)\left(m_{n}+\beta_{n}\right), \quad n=1,2, \ldots
$$

Доказательство условия В). Докажем, что

$$
\max _{1 \leqslant k \leqslant N_{n}} \mathbf{P}\left\{\left|X_{n, k}\right| \geqslant \varepsilon\right\}=\mathbf{P}\left\{\left|X_{n, 1}\right| \geqslant \varepsilon\right\} \longrightarrow 0 \quad \forall \varepsilon>0 \text { при } n \rightarrow \infty
$$

Доказательство (20) аналогично доказательству соотношения (18). Предположим противное, т. е. что выполняется неравенство $\mathbf{P}\left\{\left|X_{n, 1}\right| \geqslant\right.$ $\varepsilon\} \geqslant \gamma$ для некоторых $\varepsilon>0$ и $\gamma>0$ и всех $n$ из некоторой последовательности $\mathcal{N}$ натуральных чисел. С помошью рассуждений, которые привели нас к (18), мы придем к противоречию. Изменения в рассуждениях сводятся к замене величин $W_{n}(q)-W_{n}^{\prime}(q)$ на величины $S_{l_{n}(q)}^{(n)}$ и соотношения (19) на соотношение

$$
f_{n}(t)^{l_{n}(q)} \longrightarrow f(t) \quad \text { при } n \rightarrow \infty, n \in \mathcal{N}_{1} .
$$

Соотношение (20) позволяет воспользоваться теоремой 1 , по которой для почти всех по мере Лебега $q \in(0,1)$ последовательности случайных величин

$$
\left\{\sum_{k=1}^{l_{n}(q)}\left(X_{n, k}-\alpha_{n}\right)\right\}_{n \geqslant 1} \text { и }\left\{N_{n} \alpha_{n}\right\}_{n \geqslant 1}
$$

слабо компактны. По лемме 4 для почти всех по мере Лебега $q \in(0,1)$ числовая последовательность $\left\{l_{n}(q)\left|\alpha_{n}\right|\right\}_{n \geqslant 1}$ ограничена.

Д о с т а т ч н о с т ь. Условие В) влечет условие А), так как

$$
S_{l_{n}(q)}^{(n)}=\sum_{k=1}^{l_{n}(q)}\left(X_{n, k}-\alpha_{n}\right)+l_{n}(q) \alpha_{n}, \quad n=1,2, \ldots
$$

Достаточность условия А) следует из соотношения (15), в котором нужно положить $B_{n}=0$. Теорема доказана.

Доказате льст во те оремы 5 . Н еобходи мость. По теореме 1 (см. замечание 2) последовательность $\left\{N_{n}\left|\alpha_{n}\right|\right\}_{n \geqslant 1}$ слабо компактна. По теореме 3 последовательность $\left\{N_{n} d_{n}\right\}_{n \geqslant 1}$ также слабо компактна и, следовательно, этим свойством обладает последовательность $\left\{N_{n}\left(d_{n}+\left|\alpha_{n}\right|\right)\right\}_{n \geqslant 1}$. По теореме 4 последовательность случайных величин $\left\{S_{l_{n}(q)}^{(n)}\right\}_{n \geqslant 1}$ слабо компактна для почти всех по мере Лебега $q \in(0,1)$. Для таких $q \in(0,1)$ по лемме 2 выполняется соотношение

$$
\sup _{n} l_{n}(q) d_{n}=D(q)<\infty \text {. }
$$


Докажем, что последовательность чисел из (3) обладает требуемыми свойствами. Разделим все натуральные числа на три непересекающихся множества

$$
\begin{aligned}
& J_{1}=\left\{n: d_{n}+\left|\alpha_{n}\right|=0\right\}, \quad J_{2}=\left\{n: d_{n}=0,\left|\alpha_{n}\right|>0\right\} \\
& J_{3}=\left\{n: d_{n}>0\right\} .
\end{aligned}
$$

Если $n \in J_{1}$, то

$$
S_{k_{n}}^{(n)}=k_{n} \alpha_{n}=0, \quad \mathbf{P}\left\{\frac{N_{n}}{k_{n}}>2\right\} \leqslant \frac{1}{n+1} .
$$

Если $n \in J_{2}$, то $\left|S_{k_{n}}^{(n)}\right| \leqslant 1, N_{n} / k_{n} \leqslant N_{n}\left|\alpha_{n}\right|$ и, следовательно, последовательности $\left\{N_{n} / k_{n}\right\}$ и $\left\{S_{k_{n}}^{(n)}\right\}, n \in J_{1} \cup J_{2}$, слабо компактны. Для завершения доказательства необходимости требуется доказать, что последовательности случайных величин $\left\{N_{n} / k_{n}\right\}$ и $\left\{S_{k_{n}}^{(n)}\right\}, n \in J_{3}$, слабо компактны. Пусть $\mathscr{N}$ - произвольная последовательность натуральных чисел. Выберем подпоследовательность $\mathcal{N}_{1} \subset \mathcal{N}$ такую, чтобы $\delta_{n} \longrightarrow \delta$ гри $n \rightarrow \infty, n \in \mathcal{N}_{1}$. Возможны два случая: 1) $\delta=0$ и 2) $\delta>0$.

Пусть имеет место случай 1). По определению метрики Леви (см. $[1, \S 9$, теорема 1]) выполняется неравенство

$\mathscr{E}\left(x-\frac{3 \delta_{n}}{2}\right)-\frac{3 \delta_{n}}{2} \leqslant R_{n}(x)=\mathbf{P}\left\{N_{n}\left(d_{n}+\left|\alpha_{n}\right|\right)<x\right\} \leqslant \mathscr{E}\left(x+\frac{3 \delta_{n}}{2}\right)+\frac{3 \delta_{n}}{2}$

для всех $x$ и, следовательно,

$$
\mathbf{P}\left\{\frac{N_{n}}{k_{n}} \geqslant 1\right\} \leqslant \mathbf{P}\left\{N_{n}\left(d_{n}+\left|\alpha_{n}\right|\right) \geqslant 2 \delta_{n}\right\} \leqslant \frac{3 \delta_{n}}{2} \longrightarrow 0
$$

при $n \rightarrow \infty, n \in \mathcal{N}_{1}$. Отсюда следует, что последовательность $\left\{N_{n} / k_{n} \mid\right\}$ $n \in \mathscr{N}_{1}$, слабо компактна. Поэтому найдутся последовательность натуральных чисел $\mathscr{N}_{2} \subset \mathscr{N}_{1}$ и функция распределения $R$, такие, что

$$
\bar{R}_{n}(x)=\mathbf{P}\left\{\frac{N_{n}}{k_{n}}<x\right\} \Longrightarrow R(x) \quad \text { при } n \rightarrow \infty, n \in \mathscr{N}_{2} .
$$

Далее заметим, что $d_{n} \rightarrow 0$ в силу $(21)$, так как $l_{n}(q) \rightarrow \infty$ при $n \rightarrow \infty$. Из определения чисел $k_{n}$ следует, что

$$
k_{n} d_{n} \leqslant\left(\frac{2 \delta_{n}}{d_{n}+\left|\alpha_{n}\right|}+1\right) d_{n} \leqslant 2 \delta_{n}+d_{n} \longrightarrow 0 \quad \text { при } n \rightarrow \infty, n \in \mathscr{N}_{2} .
$$

По лемме 3 последовательность случайных величин $\left\{S_{k_{n}}^{(n)}-k_{n} \alpha_{n}\right\}$, $n \in \mathcal{N}_{2}$, сходится по вероятности к нулю. В силу (20) выполняется соотношение $\left|\alpha_{n}\right| \rightarrow 0$ при $n \rightarrow \infty$. Принимая в внимание соотношения

$$
k_{n}\left|\alpha_{n}\right| \leqslant\left(\frac{2 \delta_{n}}{d_{n}+\left|\alpha_{n}\right|}+1\right)\left|\alpha_{n}\right| \leqslant 2 \delta_{n}+\left|\alpha_{n}\right| \longrightarrow 0 \text { при } n \rightarrow \infty, n \in \mathscr{N}_{2} \text {, }
$$


мы видим, что последовательность $\left\{S_{k_{n}}^{(n)}\right\}, n \in \mathscr{N}_{2}$, слабо сходится по вероятности к нулю.

Пусть теперь имеет место случай 2). Из неравенства

$$
\frac{N_{n}}{k_{n}} \leqslant \frac{N_{n}\left(d_{n}+\left|\alpha_{n}\right|\right)}{2 \delta_{n}}, \quad n \in \mathcal{N}_{1},
$$

и слабой компактности последовательности $\left\{N_{n}\left(d_{n}+\left|\alpha_{n}\right|\right)\right\}_{n \geqslant 1}$ следует, что последовательность $\left\{N_{n} / k_{n}\right\}, n \in \mathcal{N}$, также слабо компактна. Поэтому выполняется (22) для некоторой последовательности натуральных чисел $\mathscr{N}_{2} \subset \mathscr{N}_{1}$. Расстояние Леви между функциями распределения $R$ и $\mathscr{E}$ равно числу $\delta$, так как $L\left(\bar{R}_{n}, \mathscr{E}\right) \longrightarrow L(R, \mathscr{E})$ при $n \rightarrow \infty, n \in \mathcal{N}_{2}$ и, следовательно, $R \neq \mathscr{E}$. Поэтому левая $q$-квантиль $l(q)$ функции распределения $R$ положительна на некотором множестве положительной меры Лебега. По лемме 4 найдется множество $Q \subset(0,1)$ положительной меры Лебега, такое, что

$$
\frac{l_{n}(q)}{k_{n}} \longrightarrow l(q)>0 \quad \text { при } n \rightarrow \infty, n \in \mathcal{N}_{1}, q \in Q .
$$

По теореме 4 для почти всех по мере Лебега $q \in(0,1)$ последовательность $\left\{S_{l_{n}(q)}^{(n)}\right\}_{n \geqslant 1}$ слабо компактна. Выберем $q \in Q$ и $\mathscr{N}_{3} \subset \mathcal{N}_{2}$ такие, чтобы последовательность функпий распределения $\left\{\mathbf{P}\left\{S_{l_{n}(q)}^{(n)}<x\right\}\right\}, n \in \mathscr{N}_{3}$, слабо сходилась к некоторой функции распределения $F$. По теореме непрерывности

$$
\mathbf{E} \exp \left\{i t S_{l_{n}(q)}^{(n)}\right\}=f_{n}^{l_{n}(q)}(t) \longrightarrow f(t) \text { при } n \rightarrow \infty, n \in \mathscr{N}_{3},
$$

для всех вещественных чисел $t$, где $f$-характеристическая функция $F$. По теореме Хинчина $[1, \S 24$, теорема 2] $f$ является безгранично делимой характеристической функщией. Поэтому любая положительная степень ее также является характеристической функцией. Привлекая (23), мы получим

$$
f_{n}^{k_{n}}(t)=\left(f_{n}^{l_{n}}(t)\right)^{k_{n} / l_{n}} \longrightarrow f^{1 / l}(t), \quad l_{n}=l_{n}(q), l=l(q) .
$$

По теореме непрерывности последовательность случайных величин $\left\{S_{k_{n}}^{(n)}\right\}$, слабо сходится. Таким образом доказано, что произвольные последовательности случайных величин $\left\{N_{n} / k_{n}\right\}$ х $\left\{S_{k_{n}}^{(n)}\right\}, n \in \mathcal{N} \subset J_{3}$, содержат слабо сходящиеся подпоследовательности. Необходимость доказана.

Д о с т а т очн ость. Из леммы 4 и предположения о слабой компактности последовательности $\left\{N_{n} / k_{n}\right\}_{n \geqslant 1}$ следует, что для почти всех по мере Лебега $q \in(0,1)$ выполняется соотношение

$$
\sup _{n} \frac{l_{n}(q)}{k_{n}}=E(q)<\infty
$$


и, следовательно, для тех же самых $q \in(0,1)$

$$
\lim _{x \rightarrow \infty} \sup _{n} \mathbf{P}\left\{\left|S_{l_{n}(q)}^{(n)}\right| \geqslant x\right\}=0
$$

Действительно, соотношение (24) имеет место, если последовательность случайных величин $\left\{S_{l_{n}(q)}^{(n)}\right\}_{n \geqslant 1}$ слабо компактна. Докажем это. Пусть $\mathcal{N}$ - произвольная последовательность натуральных чисел. Выберем подпоследовательность $\mathcal{N}_{1} \subset \mathcal{N}$ такую, чтобы $l_{n}(q) / k_{n} \longrightarrow l(q)$ и последовательность функций распределения $\left\{\mathbf{P}\left\{S_{k_{n}}^{(n)}<x\right\}\right\}, n \in \mathcal{N}_{2}$, слабо сходилась к некоторой функции распределения $F$. По теореме Хинчина $[1, \S 24$, теорема 2] функция распределения $F$ безгранично делима. По теореме непрерывности

$$
\mathbf{E} \exp \left\{i t S_{k_{n}}^{(n)}\right\}=f_{n}^{l_{n}(q)}(t) \longrightarrow f(t) \quad \text { при } n \rightarrow \infty, n \in \mathscr{N}_{2}
$$

для всех вещественных чисел $t$, где $f$ - характеристическая функция $F$. Из этих соотношений следует, что

$$
\begin{aligned}
\operatorname{Eexp}\left\{i t S_{l_{n}(q)}^{(n)}\right\} & =f_{n}^{l_{n}(q)}(t) \\
& =\left(f_{n}^{k_{n}}(t)\right)^{l_{n}(q) / k_{n}} \longrightarrow f^{l(q)}(t) \quad \text { при } n \rightarrow \infty, n \in \mathscr{N}_{1}
\end{aligned}
$$

и, следовательно, последовательность случайных величин $\left\{S_{l_{n}(q)}^{(n)}\right\}$, $n \in \mathscr{N}_{1}$, слабо сходится. Соотношение (24) доказано. Из (24) и равенства

$$
\mathbf{P}\left\{\left|S_{N_{n}}^{(n)}\right| \geqslant x\right\}=\int_{0}^{1} \mathbf{P}\left\{\left|S_{l_{n}(q)}^{(n)}\right| \geqslant x\right\} d q,
$$

которое можно доказать так же, как было доказано (10), следует, что

$$
\lim _{x \rightarrow \infty} \sup _{n} \mathbf{P}\left\{\left|S_{N_{n}}^{(n)}\right| \geqslant x\right\}=0
$$

По критерию (1) последовательность случайных сумм $\left\{S_{N_{n}}^{(n)}\right\}_{n \geqslant 1}$ слабо компактна. Теорема доказана.

Доказательство замечания 3 . Н е о о м о с ть. Пусть числа $k_{n}$ определены, как указано в (3). Докажем, что последовательность случайных величин $\left\{N_{n}\left(d_{n}+\left|\alpha_{n}\right|\right)\right\}_{n \geqslant 1}$ стохастически отделима от нуля. Предположим противное. Тогда некоторая ее подпоследовательность $\left\{N_{n}\left(d_{n}+\left|\alpha_{n}\right|\right)\right\}, n \in \mathcal{N}$, слабо сходится по вероятности к нулю и, следовательно,

$$
\delta_{n}=L\left(R_{n}, \mathscr{E}\right) \longrightarrow 0 \quad \text { при } n \rightarrow \infty, n \in \mathcal{N},
$$

где $R_{n}(x)=\mathbf{P}\left\{N_{n}\left(d_{n}+\left|\alpha_{n}\right|\right)<x\right\}$. Отсюда следует (см. доказательство теоремы 5), что последовательность случайных величин $\left\{S_{k_{n}}^{(n)}\right\}, n \in \mathcal{N}$, 
слабо сходится к нулю и, следовательно (см. также [5, лемму 4]), последовательность случайных сумм $\left\{S_{N_{n}}^{(n)}\right\}, n \in \mathcal{N}$, также сходится по вероятности к нулю, чего по предположению не может быть.

Обозначим $m_{n}=\left[1 /\left(d_{n}+\left|\alpha_{n}\right|\right)\right]$ и заметим, что последовательность случайных величин $\left\{N_{n} / m_{n}\right\}_{n \geqslant 1}$ слабо компактна. Докажем, что последовательность случайных величин $\left\{S_{m_{n}}^{(n)}\right\}_{n \geqslant 1}$ слабо компактна. Возьмем произвольную последовательность натуральных чисел $\mathcal{N}$. Выберем подтоследовательность $\mathcal{N}_{1} \subset \mathcal{N}$, такую, чтобы $\delta_{n} \rightarrow \delta$ и $R_{n} \Longrightarrow R$ при $n \rightarrow \infty, n \in \mathscr{N}_{1}$. Функция распределения $R$ отлична от вырожденной функции распределения $\mathscr{E}$ и, следовательно, $\delta>0$, так как $\delta_{n}=L\left(R_{n}, \mathscr{E}\right) \longrightarrow L(R, \mathscr{E})$ при $n \rightarrow \infty, n \in \mathcal{N}_{1}$. Поэтому

$$
\frac{k_{n}}{m_{n}} \longrightarrow 2 \delta \quad \text { при } n \rightarrow \infty, n \in \mathcal{N}_{1} \text {. }
$$

По теореме 5 последовательность случайных величин $\left\{S_{k_{n}}^{(n)}\right\}_{n \geqslant 1}$ слабо компактна и, следовательно, последовательность $\left\{S_{m_{n}}^{(n)}\right\}, n \in \mathcal{N}_{1}$, также слабо компактна. Таким образом доказано, что каждая последовательность $\left\{S_{m_{n}}^{(n)}\right\}, n \in \mathcal{N}$, содержит слабо сходящуюся подпоследовательность. Необходимость доказана. Доказательство достаточности можно осушествить так же, как доказательство достаточности в теореме 5. 3амечание доказано.

Доказательство те оремы 6. Н еобходи мость. Заметим, что (18) выполняется. По теореме 2 для слабой компактности последовательности случайных сумм $\left\{S_{N_{n}}^{(n)}-A_{n}\right\}_{n \geqslant 1}$ необходимо и достаточно, чтобы последовательности случайных величин

$$
\left\{\sum_{k=1}^{N_{n}}\left(X_{n, k}-m_{n}-\beta_{n}\right)\right\}_{n \geqslant 1} \text { и }\left\{N_{n}\left(m_{n}+\beta_{n}\right)-\operatorname{med}\left(N_{n}\left(m_{n}+\beta_{n}\right)\right)\right\}_{n \geqslant 1}
$$

были слабо компактными. По теореме 5 первая из этих двух последовательностей слабо компактна тогда и только тогда, когда слабо компактны последовательности $\left\{N_{n} / k_{n}\right\}_{n \geqslant 1}$ и $\left\{S_{k_{n}}^{(n)}-k_{n}\left(m_{n}+\beta_{n}\right)\right\}_{n \geqslant 1}$, где числа $k_{n}$ определены в (4). Необходимость доказана.

Д о с т а т ч н о с т ь. По теореме 5 слабая компактность последовательностей $\left\{N_{n} / k_{n}\right\}_{n \geqslant 1}$ и $\left\{S_{k_{n}}^{(n)}-k_{n}\left(m_{n}+\beta_{n}\right)\right\}_{n \geqslant 1}$; влечет слгаую компактность последовательности $\left\{S_{N_{n}}^{(n)}-N_{n}\left(m_{n}+\beta_{n}\right)\right\}_{n \geqslant 1}$. По условию последовательность $\left\{N_{n}\left(m_{n}+\beta_{n}\right)^{n}-\operatorname{med}\left(N_{n}\left(m_{n}+\beta_{n}\right)\right)\right\}_{n \geqslant 1}$ слабо компактна. Применение леммы 6 завершает доказательство теоремы.

Д ок а 3 а т ельст в о з а м е а и и я 4. Оно состоит в формальном повторении доказательства теоремы 6. См. также доказательство замечания 2. 


\section{СПИСОК ЛИТЕРАТУРЫ}

1. Гнедемко Б. В., Колмогоров $A$. Н. Предельные распределения для сумм независимых случайных величин. М.- Л.: Гостехиздат, 1949, 264 с.

.2. Круглов В. М., Королев В. Ю. Предельные теоремы для случайных сумм. М.: изд-во МГУ, 1990, 269 с.

3. Лоэв М. Теория вероятностей. М.: ИЛ, 1962, 719 с.

4. Зигель $Г$. Компактность последовательности сумм независимых величин со значениями в гильбертовом пространстве. - Литов. матем. сб., 1981, т. 21, № 4, c. $123-136$.

5. Саас Д. О классах предельных распределений для сумм случайного числа одинаково распределенных случайных величин. - Теория вероятн. и ее применен., 1972, т. XVII, в. 3, c. 424-439.

6. Korolev V. Yu., Kruglov V. M. Limit theorems for random sums of independent random variables. - Lecture Notes Math., 1993; v. 1546, p. 100-120.

7. Rosinki $J$. Weak compactness of laws of random sums of identically distributed random vectors in Banach spaces: - Colloquium Mathem., 1976, v. 35, p. 313-325.

8. Rosinski J. Shift compactness, concentration function and random sums of random vectors. - Bulletin De L'Academie Pol. Sci. Serie math. astr., 1976, v. 24, № 11, p. 1029-1033.

9. Szász $D$. Limit theorems for the distributions of the sums of a random number of random variables. - Ann. Math. Stat., 1972, v. 43, № 6, p. 1902-1913.

Поступила в редакцию 